Methods We included 177 HIV-positive MSM who completed a questionnaire regarding UAI and viral sorting practice. Viral sorting was defined as intentionally engaging in UAI on the condition that both the participant and the HIV-positive partner had an undetectable viral load, or the participant himself had an undetectable viral load with an HIV-negative/unknown casual partner. We examined whether men ever practiced intentional viral sorting since HIV diagnosis, and how often UAI with a last casual partner was the result of viral sorting. We distinguished two casual partner types: the casual sex partner (met by chance and had sex with") and the sex buddy ("contacted on a regular basis for sex but not considered a steady partner").

Results Of the 177 participants, 68\% (120/177) ever had UAI since HIV diagnosis. Of those, 44\% (53/120) ever practiced viral sorting with an HIV-positive partner and $38 \%(46 / 120)$ with an HIVnegative/unknown partner. Of all participants, 41\% (73/177) had UAI with a last casual partner. Among men who had UAI with a last HIV-positive casual partner $(n=35)$, proportions of viral sorting practice with a casual sex partner and a sex buddy were, respectively, 20\% (3/15) and 58\% (11/20), p <0.05. Among men who had UAI with an HIV-negative/unknown casual partner $(n=38)$, proportions of viral sorting with a casual sex partner and a sex buddy were, respectively, $57 \%(16 / 28)$ and $40 \%(4 / 10), p=0.47$.

Conclusions Our data suggest that viral sorting as an intentionally practiced HIV risk reduction strategy is applied relatively frequently among HIV-positive MSM with all partner types. The highest proportions were reported with HIV-positive sex buddies and HIVnegative/unknown casual sex partners. Since viral sorting is being extensively applied by MSM and most of the available data on viral load and HIV transmission risk is derived from studies on heterosexuals, future investigation should provide clear-cut indications on the effectiveness of viral sorting in lowering HIV transmission among MSM.

\section{P2-S6.08 EXAMINING RISK IN HIGH RISK “POPULATIONS: MEASURING SEXUAL BEHAVIOUR AMONG MEN WHO HAVE SEX WITH MEN"}

doi:10.1136/sextrans-2011-050108.360

\begin{abstract}
${ }^{1} \mathrm{~J}$ G Rosenberger, ${ }^{1} \mathrm{M}$ Reece, ${ }^{2} \mathrm{D}$ S Novak, ${ }^{1} \mathrm{D}$ Herbenick, ${ }^{3} \mathrm{~J} \mathrm{D}$ Fortenberry, ${ }^{1} \mathrm{~B}$ Van Der Pol. ${ }^{1}$ Indiana University, Bloomington, USA; ${ }^{2}$ Online Buddies Inc, Cambridge, USA; ${ }^{3}$ Indiana University School of Medicine, Indianapolis, USA
\end{abstract}

Purpose Due to the disproportionate impact of HIV and other sexually transmitted infections (STIs) on men who have sex with men (MSM), men who indicate same gendered sexual interactions are categorised as a priority population and often perceived as highrisk". While acknowledging the potential for increased risk among certain populations is important for disease prevention and transmission efforts, this approach broadly labels men based on a limited behavioural profile without consideration for the contextual factors of a given sexual event that influence potential disease exposure. This study sought to assess sexual behaviour among MSM in the USA during their most recent sexual event and identify factors associated with decreased risk for HIV/STI.

Methods Data were collected via an internet survey from 27,690 18-80-year old MSM. Measures included sociodemographics, recent/ lifetime sexual behaviour history, and sexual experience items.

Results Participants' median age was 39.0 years, ethnicities included white (84.5\%), Latino (6.4\%), African American (3.5\%), and most $(79.9 \%)$ identified as homosexual. Most participants reported a sexual event within the past month (86.1\%), with the majority indicating their most recent event in the past 7 days $(60.1 \%)$. While most men reported not engaging in insertive (35.2\%) or receptive (37.0\%) anal intercourse, of those who did, $46.1 \%$ used a condom and almost none reported ejaculation occurring in their or their partner's anus, $2.7 \%$ and $2.5 \%$ respectively. Among men $(24.5 \%)$ who described their sexual partner as a boyfriend or spouse, nearly half reported they and their partner had not had other sexual partners during the past 6 months $(44.7 \%)$, and the majority had been tested for STIs (61.6\%) and HIV (64.8\%).

Conclusions These data provide a large scale assessment of sexual behaviour during the most recent sexual event among MSM in the USA. Findings from this study highlight diversity in behaviours and demonstrate varying degrees of potential risk for HIV and other STIs, regardless of gender. Future prevention efforts should consider contextual components of sexual events, including partner type, HIV and STI testing patterns, and semen exposure, to more accurately develop custom risk reduction strategies.

\section{P2-S6.09 FACTORS ASSOCIATED WITH HIV SEXUAL RISK MANAGEMENT AMONG HIV-NEGATIVE MEN WHO HAVE SEX WITH MEN LIVING IN QUEBEC AND WHO USE INTERNET TO FIND SEXUAL PARTNERS}

doi:10.1136/sextrans-2011-050108.361

${ }^{1} \mathrm{M}$ C Drouin, ${ }^{2} \mathrm{~A}$ Léobon, ${ }^{3} \mathrm{~L}$ R Frigault, ${ }^{4} \mathrm{~J} \mathrm{~J}$ Lévy. ${ }^{1}$ Intitut national de santé publique $d u$ Québec, Montréal, Canada; ${ }^{2}$ CNRS: Centre national de recherche sociale, France, ${ }^{3}$ Direction de santé publique de Montréal, Canada; ${ }^{4}$ Université du Québec à Montréal, Canada

Background This presentation has 2 primary objectives. First, to describe sociodemographic, psychosocial, sociosexual, environnemental and health predictors of a HIV seroconversion risk behaviour, unprotected anal intercourse with HIV-positive or HIVunknown casual partners (UAI(+/?)), among HIV-negative MSM who live in Quebec and use Internet to find sexual partners. Second, to analyse factors associated to those predictors.

Method We use data from Net Gay Baromètre 2008, a online quantitative survey which took place on Quebec' dating websites from December 2007 to May 2008. 3718 MSM participated in this survey of whom 1794 were HIV-negative. Bivariate analysis $\left(\chi^{2} ; \mathrm{t}\right.$ test) and hierarchical regression were performed with SPSS v0.16.0 for Macintosch.

Results $14.7 \%$ of HIV-negative respondents have declared an UAI $(+/$ ?) in the past 12 months. Multivariate analysis show that homosexual identity, number of casual partners, marginal sexual practices, drug use, engagement in a couple relationship and history of a STI in the past 12 months were significant predictors associated with $\operatorname{UAI}(+/$ ?) among those respondents. Bivariate analysis show that those predictors were more often declared among respondents who live in Montreal region, are seeking sensations, are seeking partners in sex venues and are regularly seeking partners on dating websites. Conclusions This group of predictors and associated factors shows various sexual scenarios. Those sexual scenarios seem to be more frequent in certain spaces like Internet, which influence the management of sexual risks. Initiatives to prevent HIV seroconversion adapted to the reality of MSM who live in Quebec and use Internet to find sexual partners are proposed.

\section{P2-S6.10 RISKS AND ATTRIBUTABLE FRACTIONS FOR HIV INFECTION AMONG MSM AT A LGBT HEALTH CENTER: CHICAGO, 2010}

doi:10.1136/sextrans-2011-050108.362

${ }^{1}$ A Hotton, ${ }^{1} \mathrm{~B}$ Gratzer, ${ }^{2} \mathrm{C}$ Estrich, ${ }^{2} \mathrm{~S}$ D Mehta. ${ }^{1}$ Howard Brown Health Center, University of Illinois, Chicago School of Public Health, Chicago, USA; ${ }^{2}$ University of Illinois Chicago, School of Public Health, Chicago, USA

Background Continued increases in HIV among men who have sex with men (MSM) underscore the need for intervention strategies that target those at highest risk of infection. 